Provided for non-commercial research and education use. Not for reproduction, distribution or commercial use.

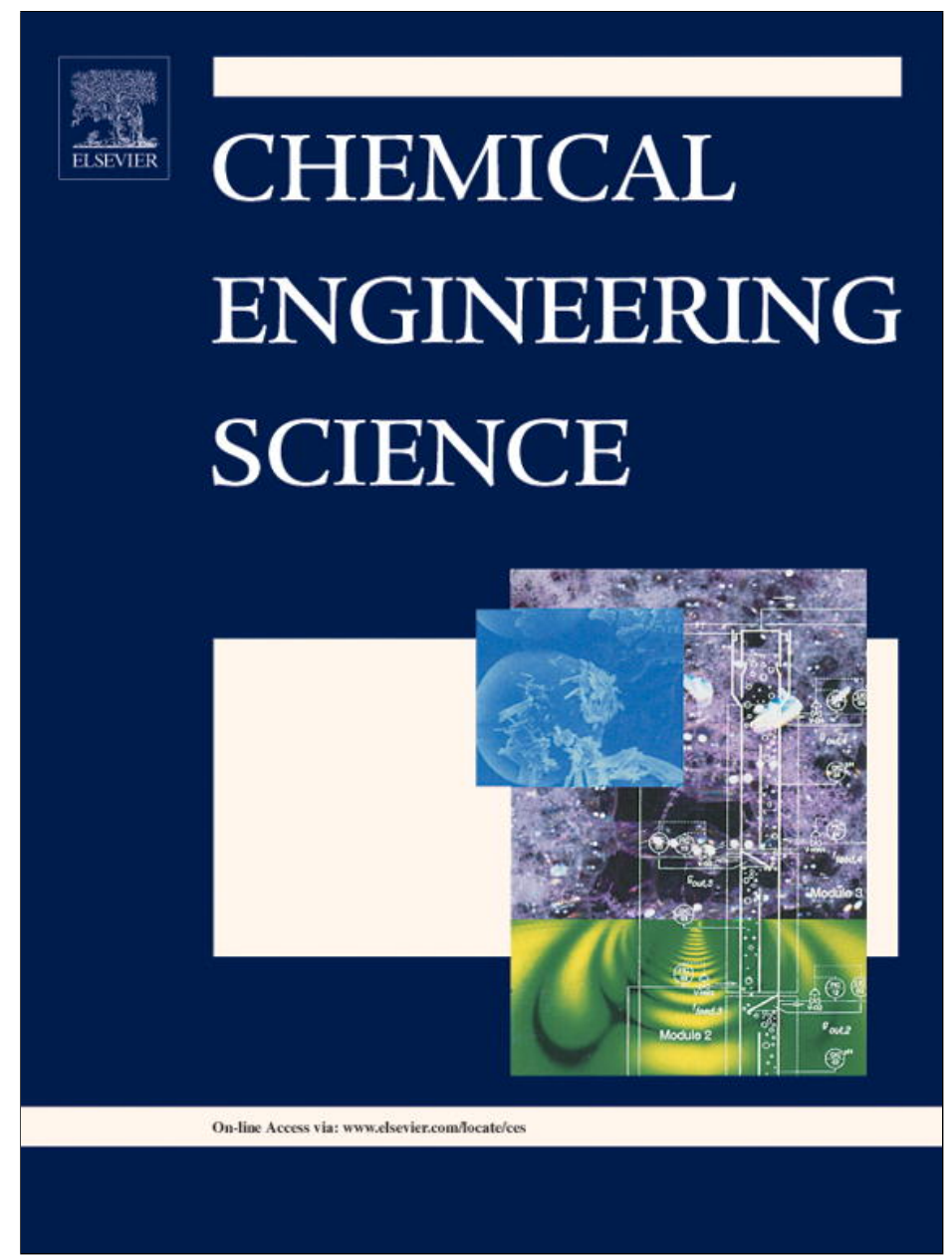

(This is a sample cover image for this issue. The actual cover is not yet available at this time.)

This article appeared in a journal published by Elsevier. The attached copy is furnished to the author for internal non-commercial research and education use, including for instruction at the authors institution and sharing with colleagues.

Other uses, including reproduction and distribution, or selling or licensing copies, or posting to personal, institutional or third party websites are prohibited.

In most cases authors are permitted to post their version of the article (e.g. in Word or Tex form) to their personal website or institutional repository. Authors requiring further information regarding Elsevier's archiving and manuscript policies are encouraged to visit:

http://www.elsevier.com/copyright 


\title{
The functionalization of carbon nanotubes using a batch oscillatory flow reactor
}

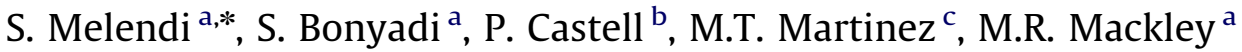 \\ a Department of Chemical Engineering and Biotechnology, University of Cambridge, New Museum Site, Cambridge CB2 3RA, UK \\ b Nanozar SL, 50018 Zaragoza, Spain \\ ${ }^{\mathrm{c}}$ Department of Carbon Nanostructures and Nanotechnology Group, Instituto de Carboquímica-CSIC, 50018 Zaragoza, Spain
}

H I G H L I G H T S

- An OFR was designed to scale up CNT functionalization with PPS compatible groups.

- Oscillatory conditions were effective and uniform mixing maintained in the reactor.

- Successful functionalization of CNT was achieved.

- CNT flocs formation was found not prevent the uniform CNT functionalization.

\section{A R T I C L E I N F O}

\section{Article history:}

Received 21 May 2012

Received in revised form

27 August 2012

Accepted 6 September 2012

Available online 13 September 2012

\section{Keywords:}

Oscillatory flow reactor

Chemical reactors

Carbon nanotubes functionalization

Mixing

Design

Scale-up

\begin{abstract}
A B S T R A C T
This paper describes an efficient method for the functionalizing of multi-walled carbon nanotubes (MWCNT) using oscillatory flow mixing (OFM). A 31 batch oscillatory flow reactor (OFR) was designed and constructed for pilot scale functionalization of MWCNT in order to potentially improve their compatibility within a thermoplastic polyphenylene sulphide (PPS) matrix. The OFM batch reactor consisted of a jacketed cylindrical vessel with a vertical axial oscillator that contained a series of baffled mixing plates. MWCNTs dispersed in dimethylformamide (DMF) were introduced into the reactor and a two stage reaction for functionalizing MWCNTs with PPS compatible groups was carried out under oscillation of baffles at elevated temperatures. Fluid mixing observations in the reactor showed that MWCNTs formed a uniform dispersion of aggregated flocs before and during the functionalization reaction. On completion of the reaction and cessation of the oscillation, the aggregated flocs of MWCNT rapidly sedimented at the bottom of the reactor; hence could be collected as a concentrated mass thereby facilitating the separation of functionalized MWCNTs from the solvent. The functionalized MWCNTs were dried and then characterized by transmission electron microscopy, infrared spectroscopy as well as thermal gravimetric analysis in order to investigate the extent of MWCNT functionalization. The characterization results confirmed the effective and relatively uniform functionalization of the MWCNTs despite formation of aggregates, indicating that OFM provides a viable approach for functionalizing MWCNTs.
\end{abstract}

Crown Copyright (c) 2012 Published by Elsevier Ltd. All rights reserved.

\section{Introduction}

Since the discovery of carbon nanotubes (CNTs) (Iijima, 1991), there has been considerable interest in how these nano scale very high aspect ratio carbon nanostructures can be used to enhance material properties. CNTs possess a combination of unique properties that make them potentially attractive for high-performance composite and electric conductivity application. See, for example, Saito (1997), Tans et al. (1998), Calvert (1999), Ramamurthy et al.

\footnotetext{
*Corresponding author. Tel.: +44 7412077279; fax: +44 1223334796 .

E-mail addresses: smelendi@gmail.com,sm876@cam.ac.uk (S. Melendi).
}

(2004, Kim et al. (2005), Coleman et al. (2006) Lau et al. (2006), Díez-Pascual et al. (2011). The high elastic modulus and conductivity properties of CNTs offer exciting opportunities for advanced composite materials such as polyphenylene sulphide (PPS) which is a semi-crystalline polymer with application as a high temperature engineering matrix material in composites that has excellent high temperature creep resistance, friction properties, chemical resistance, and inherent flame retardancy (Platt, 2003).

The incorporation of CNTs into a thermoplastic polymer such as PPS presents certain difficulties such as chemical bonding and CNTs tend to aggregate during processing, which could lead to a poor dispersion in the composite and limits the efficiency of CNTs within polymer matrices (see, for example, Qiam et al. (2000)). The effective 
incorporation of CNTs in a polymer matrix strongly depends on their disentanglement and dispersion (Huang et al., 2006), straightening (Shao et al., 2009), alignment (Jin et al., 1998; Thostenson and Chow, 2002; Gojny et al., 2004) and also high interfacial bonding (Wood et al., 2001; Deng et al., 2007). Therefore to maximize the effectiveness of CNTs as reinforcement agents in composites, the CNTs should benefit by having good functional interaction or even chemical bonding to the matrix and the nano fillers also need to be well dispersed in order to maximize the transference of properties from the filler to the polymer matrix (Ajayan et al., 2000).

In order to improve the dispersion of CNTs in a polymeric matrix, different approaches have been examined, such as optimum blending, in situ polymerization and chemical functionalization (Xie et al., 2005). In relation to thermoplastics, the introduction of grafted polymers similar to that of the matrix, onto the surface of CNTs has been considered (Tasis et al., 2006). The nature of the grafted structure is desired to be compatible to the polymeric matrix where it has to be incorporated and therefore the selection of suitable grafted groups is crucial to achieve the desired bonding within the matrix. After covalent bonding of grafted molecules onto the CNTs surface, the resulting materials could be expected to display both improved dispersion and adhesion to the matrix leading to enhanced properties of the composite. A problem with grafting strategies is that the chemistry involved in the grafting processes can result in very timeconsuming steps which present increasingly more challenging conditions with scale up from laboratory to pilot scale.

To achieve successful functionalization chemistry, especially when dealing with CNTs, one of the essential aspects is the efficiency of mixing within the reactor and the recovery of the functionalized CNTs from the reaction solvent. The most broadly used batch reactor is the stirred tank reactor, where a centrally mounted impeller generates fluid mixing (see, for example, Nienow (2010)). In these reactors, mixing is a very strong function of position with high shear at the impeller and lower levels of shear near outer wall. As a result, it is often difficult to scale up stirred tank reactors and predict the uniformity of mixing within the reactor (Perry, 1984).

An alternative to stirred tanks are oscillatory flow mixing (OFM) reactors. OFM is a specialized type of fluid mixing generated by axial oscillation of the bulk fluid in a tubular device containing periodically spaced constrictions (baffles), (see, for example, Harvey et al. (2001)). The resulting flow is characterized by complex eddies, formed as a result of fluid passing through constrictions, which potentially provides an improved fluid mixing within the reactor. Furthermore, OFMs provide the advantage of easier scalability in comparison with the stirred tank vessels (Smith and Mackley, 2006).

This paper is concerned with the design, development and validation of an oscillatory flow pilot scale reactor system built to evaluate the feasibility of an efficient CNT functionalization and the production of adequate quantities of functionalized CNTs for subsequent incorporation within a PPS matrix in order to potentially improve the mechanical properties of the polymer. The chemical reaction procedure used in this paper for the CNT functionalization with PPS compatible groups have recently been reported using a small scale laboratory unit (Gonzalez-Dominguez et al., 2012).

\section{Background on fluid mechanics within the OFR}

The fluid mechanics within a baffled vessel is controlled by the geometrical configuration of the baffles as well as the oscillatory conditions including amplitude and frequency of oscillation, which lead to the formation of different flow regimes within the reactor (see, for example, Howes (1988), Harvey et al. (2001)).
The nature of the flow is normally characterized by two dimensionless groups, namely oscillatory Reynolds number $\left(R e_{o}\right)$ and the Strouhal number $(S t r)$. $R e_{o}$ describes the intensity of oscillation as

$R e_{o}=x_{0} f d_{e} \rho / \mu$

where $x_{o}$ is the centre to peak amplitude for a sinusoidal oscillation, $f$ is the frequency of oscillation, $\rho$ is the density, $\mu$ is the viscosity, and $d_{e}$ is the effective diameter. The effective diameter takes into account the multi-orifice geometry, and it is equivalent to the total baffle diameter $(d)$ divided by the number of orifices $(n)$ (Smith, 1999):

$d_{e}=(d / n)^{1 / 2}$

Strouhal number is defined as

Str $=d_{e} / 4 \pi x_{o}$

and represents the amplitude ratio of oscillation.

Depending on the range of the oscillatory Reynolds number, different flow regimes can be obtained within the reactor. The onset of eddy formation has been found to be at Re oscillatory number in the range of 50-100 (Neves-Saraiva, 1998), and chaotic flow regimes have been reported to occur at $R e$ oscillatory number above 300 (Howes, 1988). Above these fluid mechanical conditions, an efficient mixing flow can be achieved within each inter-baffle region, which results in essentially uniform mixing throughout the reactor with a controlled intensity produced from a suitable choice of the amplitude and frequency of oscillation.

\section{Development of oscillatory flow reactor}

\subsection{Reactor design}

Batch oscillatory flow reactors have been reported before (see, for example, Ni and Mackley (1993)) and a Company Nitech Solutions was formed in 2003 to specifically develop both batch and continuous flow OFM technology (http://www.nitechsolutions.co.uk). Different reactions require different size specifications and design, and in the case of the CNT reactor it was essential that the reactor would fit inside a laboratory fume cupboard because of safety issues relating to chemicals, solvents and CNTs themselves. An oscillatory flow pilot scale reactor (OFR) system was designed and developed for the purpose of producing adequate quantities of functionalized CNTs for subsequent incorporation within a PPS thermoplastic matrix. This reactor consisted of a jacketed glass vessel, a set of baffles connected to a linear actuator and an oil circulator.

OFRs can be operated in different configurations, horizontal and vertical. In this research, a vertical configuration was adopted in order to benefit from the advantage of subsequently removing the CNTs by gravity sedimentation and also overcoming sealing issues. The batch OFR consisted of a jacketed reaction vessel of $104 \mathrm{~mm}$ internal diameter, $476 \mathrm{~mm}$ and 31 capacity (Fig. 1A and B). The height of the reactor was limited to $476 \mathrm{~mm}$ due to space limitations inside laboratory and the reactor diameter was limited to $100 \mathrm{~mm}$ due to some constructional limitations. Both the glass reactor vessel and reactor stand were supplied by Radleys (Essex, UK).

Fig. 1C shows the schematic diagram of the baffle disc used in the OFR. The discs were machined of stainless steel into dimensions of 96-mm diameter, 2-mm thickness and coupled using stainless steel studding. The baffle discs were composed of 12 orifices each having a diameter of $13 \mathrm{~mm}$ giving a constriction ratio of $78 \%$ by area as previously this value was successfully used in batch reactions ( $\mathrm{Ni}$ and Mackley, 1993). The baffle constriction percentage is the percentage reduction of cross-sectional area in the tube provided by the baffle and is usually in the range of $50-80 \%$ based on a compromise between minimizing frictional losses and maximizing the mixing effect (Brunold et al., 1989). The inter-baffle spacing is vital for 

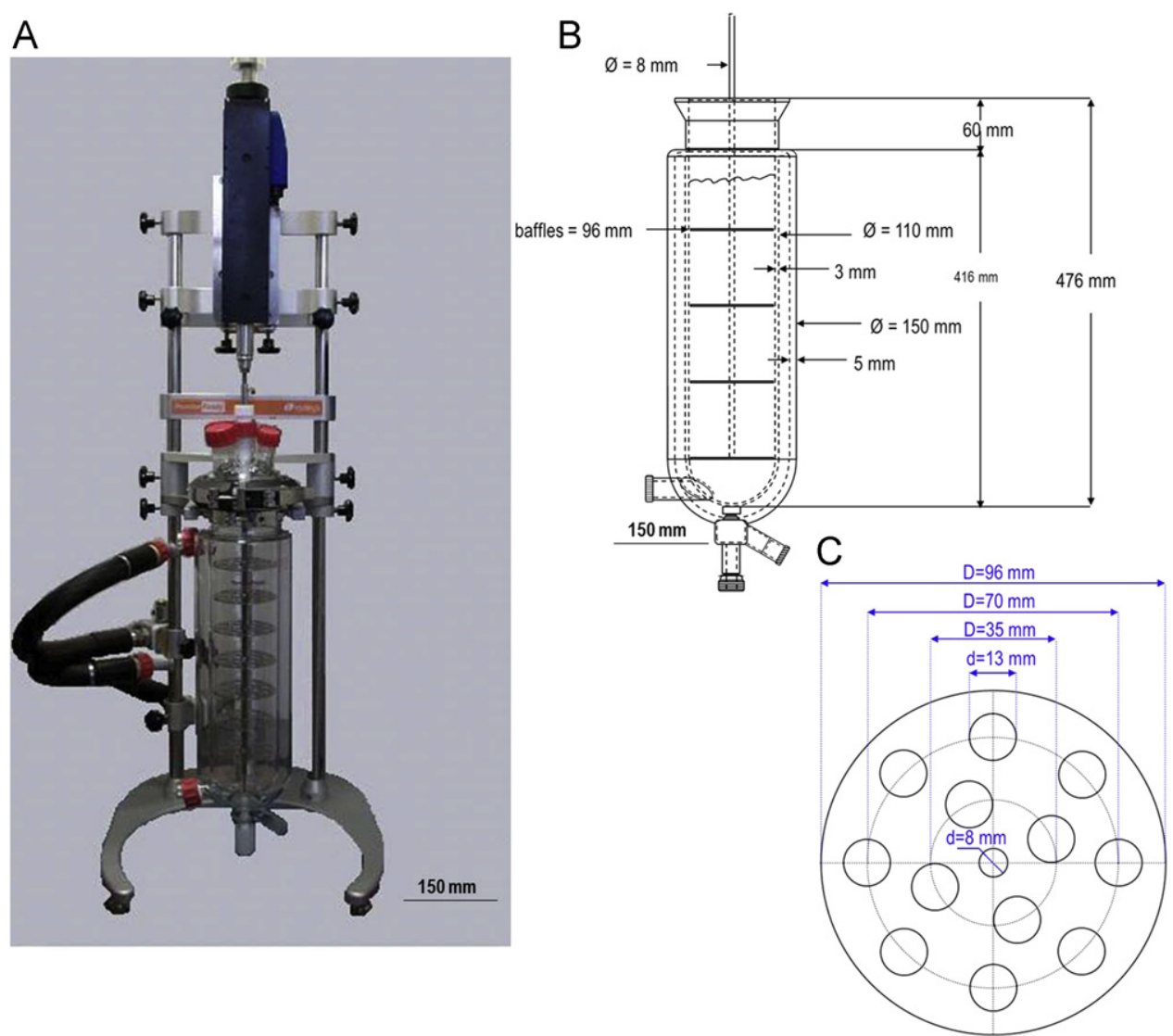

Fig. 1. 31 oscillatory flow reactor (OFR). (A) Photograph of reaction vessel connected to the oscillation system. (B) Dimensions of the vessel. (C) Dimensions of the baffles.
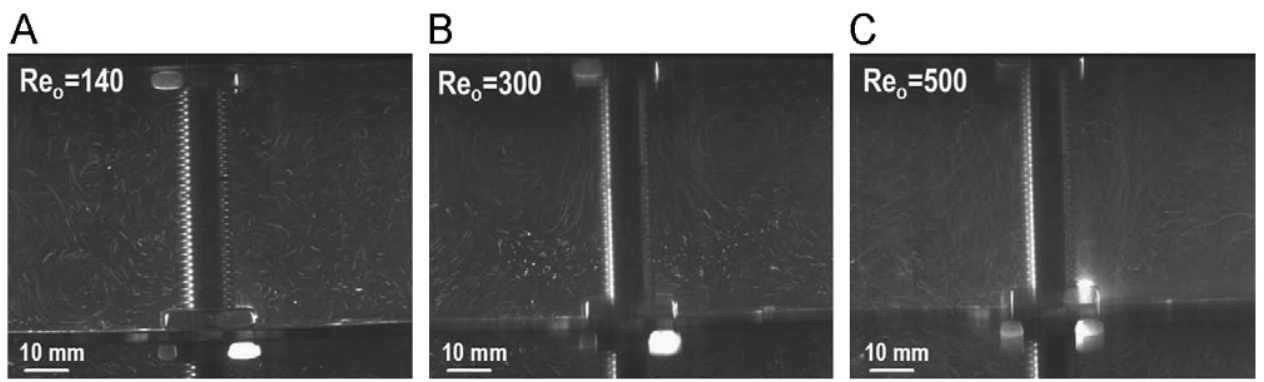

Fig. 2. Visualization of flow in the OFR. Tracer PS $125 \mu \mathrm{m}$. (A) Oscillatory conditions: frequency $1 \mathrm{~Hz}$, amplitude centre to peak $5 \mathrm{~mm}$ (approx. Re $e_{o}=140$ ). (B) Oscillatory conditions: frequency $1 \mathrm{~Hz}$, amplitude centre to peak $10.5 \mathrm{~mm}$ (approx. $R e_{o}=300$ ). (C) Oscillatory conditions: frequency $2 \mathrm{~Hz}$, amplitude centre to peak $8.5 \mathrm{~mm}\left(\right.$ approx. $R e_{o}=500$ ).

a good mixing within oscillatory flow and is related to the effective diameter of the tube as $1.5 d_{e}$ (Smith, 1999). Therefore, considering the maximum height of the tube and a required excess space at the bottom and top of the reactor, seven discs were attached to a central stainless steel rod of 8-mm diameter.

In terms of oscillatory flow mixing, there were different methods employed to create the oscillations. In principle, it is possible to oscillate the fluid or the baffles. Generally, it is technically more straightforward to oscillate the baffles than the fluid as the latter can result in issues such as creating a good seal around the piston whilst minimizing friction. Therefore, it was decided to generate the sinusoidal oscillation of the baffles in a reciprocating plate column.

In order to create the sinusoidal oscillation of the baffles a ServoTube STA2510S linear actuator (Copley Controls, UK), shown at the top of Fig. 1A, was used to generate the mixing, and this was controlled by a Xenus XTL amplifier (Copley Controls, UK) which was linked to a PC running CME2 software (Copley Controls, UK). The device was capable of producing $0-20 \mathrm{~mm}$ centre-to-peak displacements and $0-3 \mathrm{~Hz}$.
The reactor was maintained at the reaction temperature by circulating silicon oil through the jacket of the vessel using a Huber CC302 oil circulator.

\subsection{Flow validation}

Prior to the functionalization experiments the effect of oscillatory mixing parameters (frequency and amplitude) on the flow geometry in the reactor was investigated by fluid flow observation. This was carried out to better understand the dynamics of mixing within the OFR and to establish good mixing conditions for functionalizing carbon nanotubes.

Visualization of flow was carried out using a QICAM Fast 1394 camera with a "LEICA mono zoom 7" lens. PS $125 \mu \mathrm{m}$ particle size tracer particles were added into the fluid in order to enhance the fluid streamline observations.

Fig. 2 shows the nature of the mixing obtainable in the OFR at different $R e_{0}$. Fig. 2 A shows the formation of smaller eddies subject to soft oscillatory conditions within the reactor at low $R e_{o}$ around 
140. By increasing the $R e_{o}$ up to 300 , more intense eddy activity was seen as shown in the single photo Fig. 2B, although movie clips show the effect very much more clearly. At $R e_{o}$ numbers above 300 , the flow regime becomes more chaotic and produced intense mixing as shown in Fig. $2 \mathrm{C}$ corresponding to $R e_{o}=500$. The flow behaviour observed during these experiments was consistent with those reported by Ni and Mackley (1993), Harvey et al. (2001).

Based on the flow observations presented in Fig. 2 and the previous reports on batch reactions using OFR (Ni and Mackley, 1993), it is clear that $R e_{o}$ numbers above 300 potentially provide a suitable mixing conditions to improve the reaction performance within the OFR. Hence, $R e_{o}=300$ was adopted as the working $R e_{o}$ (corresponding to $1 \mathrm{~Hz}$ frequency and $10.5 \mathrm{~mm}$ centre-to-peak amplitude) to carry out CNT functionalization experiments in order to provide a good quality of mixing.

\section{Functionalization reaction of CNTs with PPS compatible groups}

\subsection{Starting materials and functionalization reaction}

CNTs, NANOCYL NC7000 MWCNTs were sourced from Nanocyl (Sambreville, Belgium) and these CNTs were produced by the catalytic carbon vapour deposition (CCVD). According to the manufacturer the selected MWCNTs had an average diameter of $9.5 \mathrm{~nm}$ and a length of $1.5 \mu \mathrm{m}$. Their purity was higher than $90 \%$ and their BET surface area about $312.5 \mathrm{~m}^{2} / \mathrm{g}$ (www.nanocyl.com).

The MWCNTs functionalization procedure used in this paper was based on the in situ formation and subsequent reaction of aryl diazonium salts, based on the work of Bahr and Tour (2001). The first step of the small lab scale process (reaction 1) is summarized in Fig. 3 which consisted of a diazonium salt lateral functionalization of the MWCNTs to produce the intermediate chloroanyline grafted MWCNTs (Gonzalez-Dominguez et al., 2012). The second part of the functionalization (reaction 2) involved grafting and subsequent growth of PPS chains onto the surface of the intermediate product produced in reaction 1 (Fahey and Ash, 1991; Park et al., 1992). Reaction 2 is summarized in Fig. 4. The optimized total duration and temperature conditions of each step at the small laboratory scale were $4 \mathrm{~h}$ for reaction 1 at $60{ }^{\circ} \mathrm{C}$ (Fig. 3) and $2 \mathrm{~h}$ for reaction 2 at $200{ }^{\circ} \mathrm{C}$ (Fig. 4) (Gonzalez-Dominguez et al., 2012). The complete reaction carried out at a small laboratory scale has been described elsewhere (Gonzalez-Dominguez et al., 2012). All reactions carried out using the pilot laboratory OFR reactor used dimethylformamide (DMF) as a solvent.

The initial protocol developed to carry out the functionalization reaction in the pilot laboratory scale OFR was a two-step protocol which involved washing and filtration of the intermediate product after reaction 1 in addition to the washing and filtration of the final product in order to remove the excess of reactants. These two processes in this reaction are very timeconsuming and could create scale-up difficulties. The washing and filtration of the final product is a necessary and unavoidable step in the process but it was an aspect of this study to investigate the feasibility of eliminating the washing and filtration of the intermediate product without sacrificing the reaction efficiency. As a result a one-step protocol was developed in which after $4 \mathrm{~h}$ of reaction 1 at $60{ }^{\circ} \mathrm{C}$ the reactor temperature was raised to $200{ }^{\circ} \mathrm{C}$ and reactants for reaction 2 were added to the reactor and the reaction continued for a further $2 \mathrm{~h}$. In this way, the washing and filtration of the intermediate product was avoided. The washing and filtration processes were carried out using Omnipore Membrane filters $1.0 \mu \mathrm{m}$ coupled with a vacuum pump.

CNT concentration is another key parameter which can affect the functionalization reaction. It is known in the literature (Vaisman et al., 2006) that CNTs are more likely to form aggregates at higher CNT concentrations, which could lower the quality of CNT dispersion and hence the reaction efficiency. The laboratory scale CNT functionalization developed by GonzalezDominguez et al. was based on $0.15 \mathrm{wt} \% \mathrm{CNT}$ initial concentration (Gonzalez-Dominguez et al., 2012); however, this concentration is far too low for large-scale pilot scale CNT functionalization purposes. As a result, it is desirable to carry out functionalization reactions at higher CNT concentrations. Therefore, another aspect of this research was to investigate whether the CNT functionalization could be carried out at higher concentrations ( $1 \mathrm{wt} \%$ as an example) without sacrificing the reaction efficiency.

\subsection{Sedimentation of CNT aggregates after reaction}

It was discovered that the oscillatory flow reactor produced a uniform dispersion of aggregated CNTs flocs. This result was unexpected, as was initially hoped that the uniform spatial mixing created by the OFM would produce a uniform unaggregated dispersion of CNTs. Dispersion of CNTs in organic solvents is notoriously difficult and the only work known where complete dispersion is spontaneously formed is that carried out at Rice University using super acids as a solvent (Davies et al., 2004). The as-received CNTs used in the OFM experiments were in the form of a powder and as the CNT was progressively added to the DMF, small aggregates of CNT appeared. These can be of the size range of microns to millimetres. When subject to OFM, the aggregates appear to flocculate to form larger aggregated flocs in the 0.1$1 \mathrm{~mm}$ range. The mechanism for this is not clear; but presumably the collision of the small aggregates with the larger ones creates a dispersion of CNT aggregated flocs. Once formed, the aggregated flocs remained stable. At first sight, this might appear to be undesirable in relation to functionalization however as this paper will show functionalization was effective. The interrogation of the CNT suspension after cessation of the oscillation (Fig. 5) revealed the existence of large flocs, quickly settling in the reactor. The rapid sediment of the large flocs within the solvent provided an effective separation mechanism and avoided having to use pressure-driven ultrafiltration.

\section{Isophentyl nitrit}
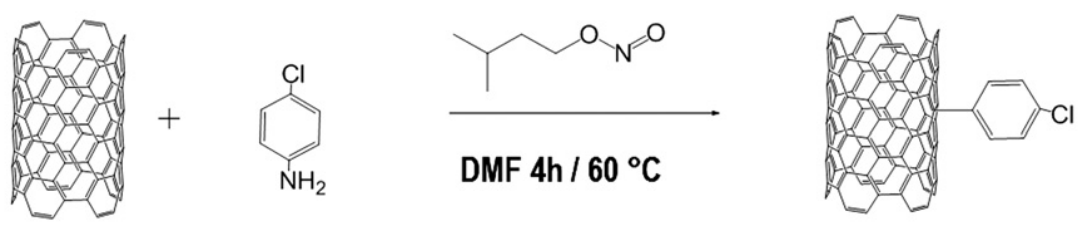

\section{MWCNT 4-chloroanline}

Chloroanyline grafted MWCNTs

Fig. 3. Grafting of chloroanyline on the surface of the MWCNTs using a diazonium salt reaction. 


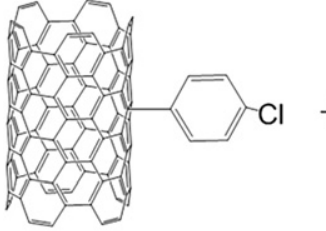

Chloroanyline grafted
MWCNTs
Sodium sulfide<smiles>Clc1ccc(Cl)cc1</smiles>

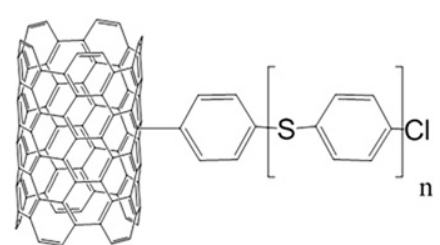

Functionalized MWCNTs

Fig. 4. Anchoring of PPS compatible chains on the surface of the modified MWCNTs.

A

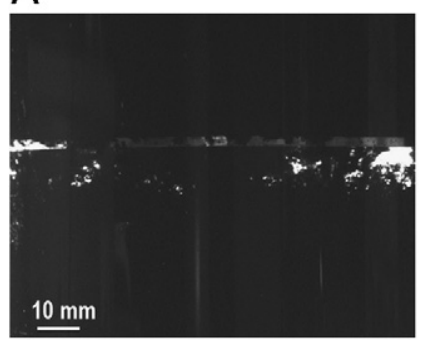

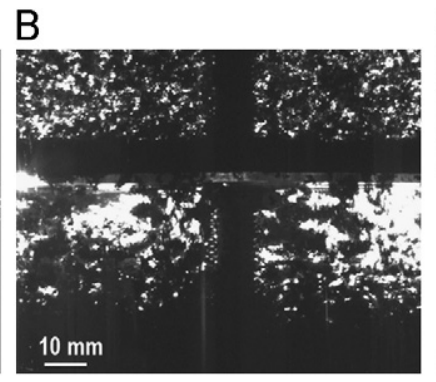

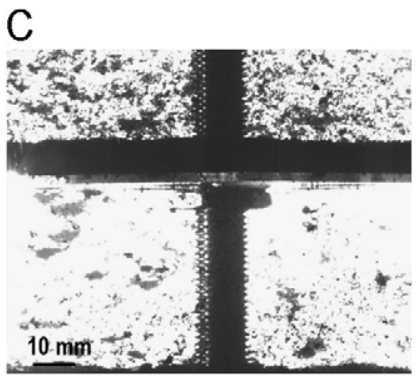

Fig. 5. FCNTs sedimentation after reaction. (A) $10 \mathrm{~s}$ after the oscillation stops. (B) $60 \mathrm{~s}$ after the oscillation stops. (C) $120 \mathrm{~s}$ after the oscillation stops.
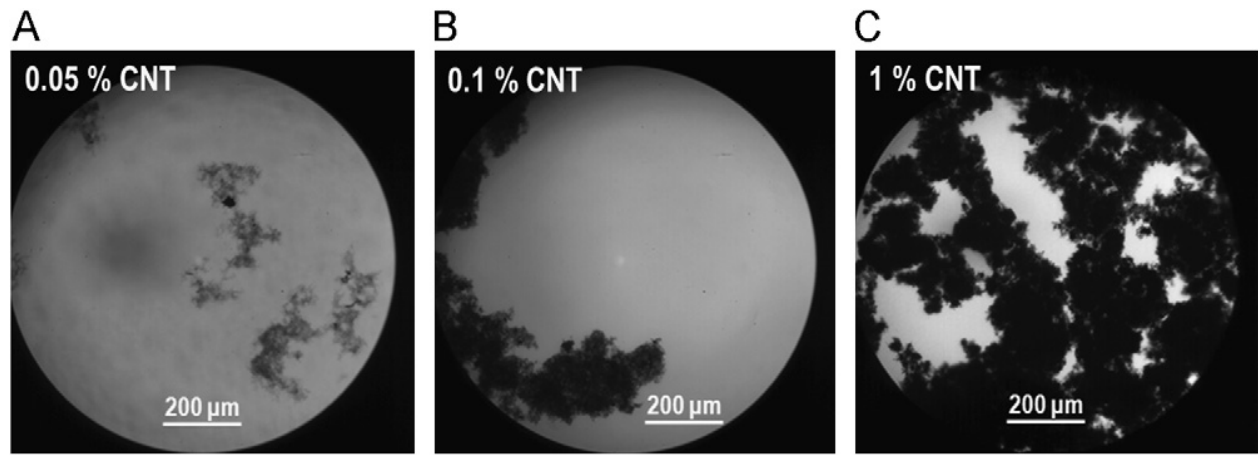

Fig. 6. Micrographs of CNT dispersions in DMF sonicated by means of a bioruptor for $1 \mathrm{~h}$. (A), (B) and (C) corresponding to different sample concentrations 0.05 wt\%, $0.1 \mathrm{wt} \%$ and $1 \mathrm{wt} \%$.

The average rate of flocs sedimentation was estimated to be $1.7 \mathrm{~mm} / \mathrm{s}$ corresponding to an average sedimentation time of $4 \mathrm{~min}$. A wide range of sedimentation velocities were observed due to the presence of a wide distribution of flocs size ranging from a few microns up to approximately $10 \mathrm{~mm}$ as shown by the settling observation images in Fig. 5.

The formation of aggregates in suspensions especially at high CNT concentrations is an ongoing issue which is not unique to the developed OFM reactor. For example, Fig. 6 shows the formation of large aggregates in suspensions even with very low CNT concentration despite the fact that they were treated for $1 \mathrm{~h}$ with a bioruptor which is a powerful ultrasound agitator (www.diagenode.com).

\subsection{Extent of reaction validation and product characterization}

The product obtained from the washing and filtration of the FCNT suspension was dried in an oven overnight at $120^{\circ} \mathrm{C}$. The dried FCNTs were in the form of aggregates and less powdery compared with the initial untreated CNTs provided by Nanocyl.

In order to confirm the effectiveness and the extent of the functionalization process and to compare the efficiency of the reactions carried out in one and two steps, the OFR processed functionalized carbon nanotubes were analysed by transmission electron microscopy (TEM), Fourier transform infrared (FTIR) spectroscopy and thermogravimetric analysis (TGA).

Transmission electron microscopy (TEM) micrographs were taken with a JEOL-2000 FXII electron microscope, working at $200 \mathrm{kV}$ and with $0.28 \mathrm{~nm}$ point-to-point resolution. The samples were prepared by drop casting several drops of $N$-methylpyrrolidone (NMP) suspension on a carbon-coated copper grid. Fourier transform infrared (FTIR) spectra were obtained with a Bruker Vertex 70 instrument. All of the samples were prepared as pellets using spectroscopic grade $\mathrm{KBr}$. Thermo gravimetric analysis (TGA) was performed under nitrogen atmosphere $(100 \mathrm{~mL} / \mathrm{min}$ flow) and registered with a SETARAM Setsys Evolution $16 / 18$ device at a heating rate of $10{ }^{\circ} \mathrm{C} / \mathrm{min}$ and temperatures ranging from room temperature to $900{ }^{\circ} \mathrm{C}$.

The TEM images in Fig. 7 show the presence of rough layers covering the MWCNTs processed through one- and two-step functionalization reactions. The presence of these rough layers suggests the successful grafting of PPS polymeric groups onto the surface of MWCNTs. These images also suggest that the formation of the covering layers almost occurred along the whole length of the MWCNTs although the thickness of the covering polymeric layers was not uniform. The grafted chains are visible on the surface as a thin layer or as globules approximately of $2-3 \mathrm{~nm}$. All the observed micrographs showed similar degree of functionalization. Thus, we can conclude that the functionalization was homogeneous. This 
A
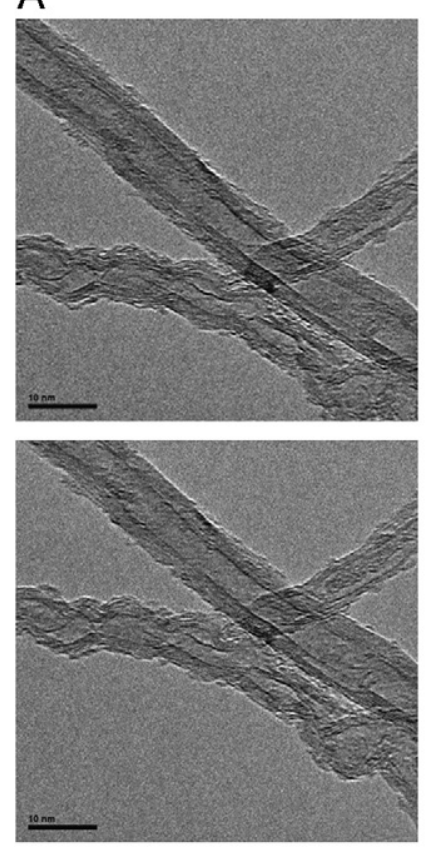

B
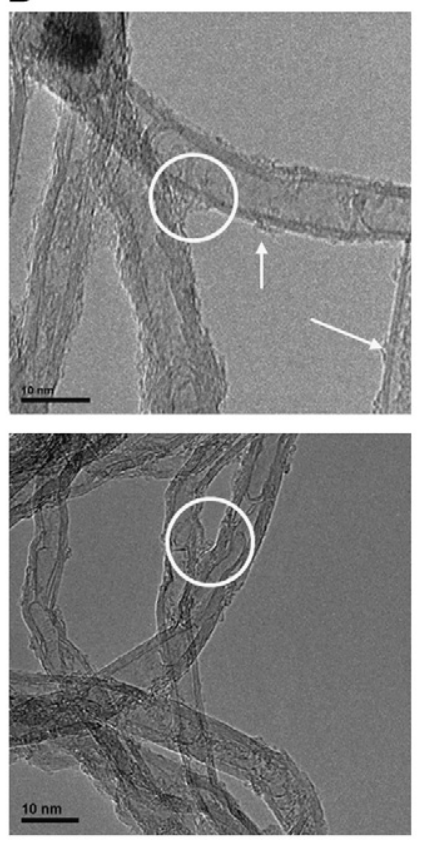

C
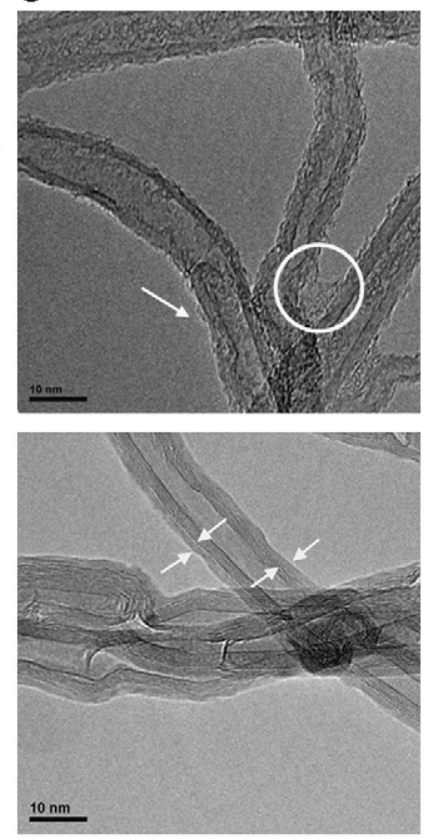

Fig. 7. TEM micrographs of CNTs (A) Untreated CNTs; (B) FCNTs from two stage OFR reaction (C) FCNTs from single stage OFR reaction.

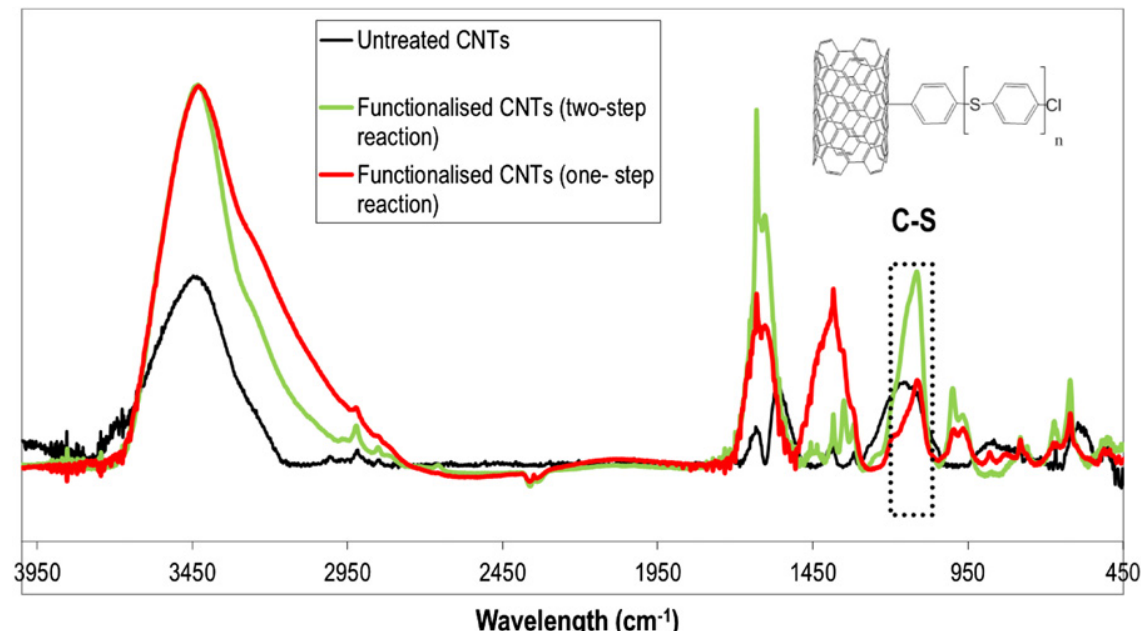

Fig. 8. FTIR spectra of the functionalized CNTs in two-step and in a single step reaction.

further suggests that formation of flocs inside the reactor did not significantly prevent the functionalization of the CNTs in the interior region of the aggregates. This could be due to a rather open structure of the flocs, which suggests the reactants can access all the CNTs in each individual floc and react with them.

The presence of the PPS groups on the MWCNTs surface was further confirmed by the FTIR spectroscopy. For both one- and two-step functionalized CNTs, a peak at $1110 \mathrm{~cm}^{-1}$ corresponding to the $\mathrm{C}-\mathrm{S}$ bond was detected in the spectra, shown in Fig. 8, which is the characteristic of the polymer chains anchored to the surface of the MWCNTs. It can be observed that the intensity of the band at $1100 \mathrm{~cm}^{-1}$ is less intense in the bare CNTs indicating that grafting of the molecules causes their appearance. The band at $1450 \mathrm{~cm}^{-1}$ could be assigned to the aromatic C-C stretching of diarylsulphur groups, observed in PPS (Tsuchida et al., 1990).

TGA analysis was performed in order to investigate the nature of bonding between the PPS groups and the MWCNTs and also estimate and compare the yield of functionalization in one- and twostep functionalization reactions. At least three different TGAs were registered for each sample in order to assure the reproducibility of the measurements. The yield of functionalization is defined as the weight percentage of the bonded polymeric groups in relation to the overall weight of the functionalized MWCNTs.

Fig. 9 compares the TGA results obtained for the pure PPS and untreated CNTs versus one-step and two-step functionalized CNTs, and Fig. 10 shows the derivative peaks. It can be seen that the untreated CNTs showed no weight loss over the whole temperature range tested, while the functionalized CNTs lost weight by increasing the temperature. It can also be seen that the rate of weight loss as a function of temperature was rather small up to temperatures around $500{ }^{\circ} \mathrm{C}$ but significantly increased at temperatures above this value. The initial losses below $120^{\circ} \mathrm{C}$ are due to solvents adsorbed in the surface of the CNTs during the functionalization and washing processes.

Based on the TGA result of the pure PPS, $500{ }^{\circ} \mathrm{C}$ can be considered as the temperature at which PPS starts chemical degradation. This suggests that the weight loss observed at temperatures lower than $500{ }^{\circ} \mathrm{C}$ in the TGA results of functionalized CNTs could be related to 


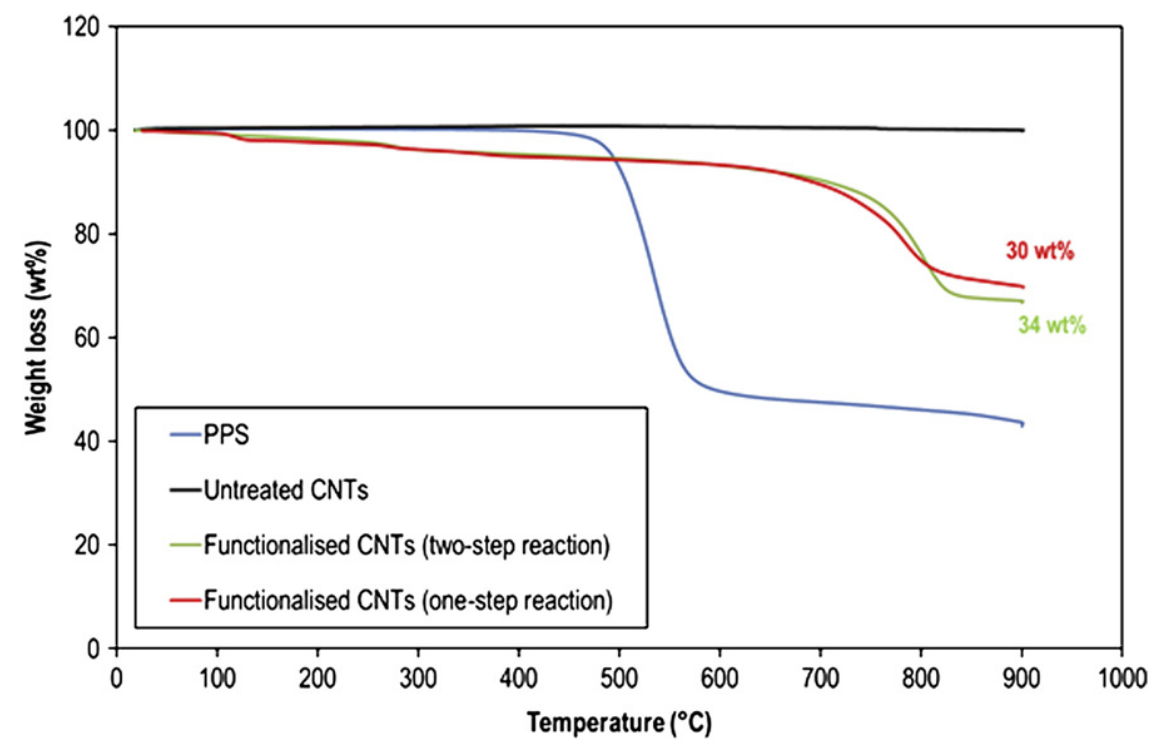

Fig. 9. TGA results of the pure PPS, untreated CNTs and functionalized CNTs in two-step and in a single step reaction.

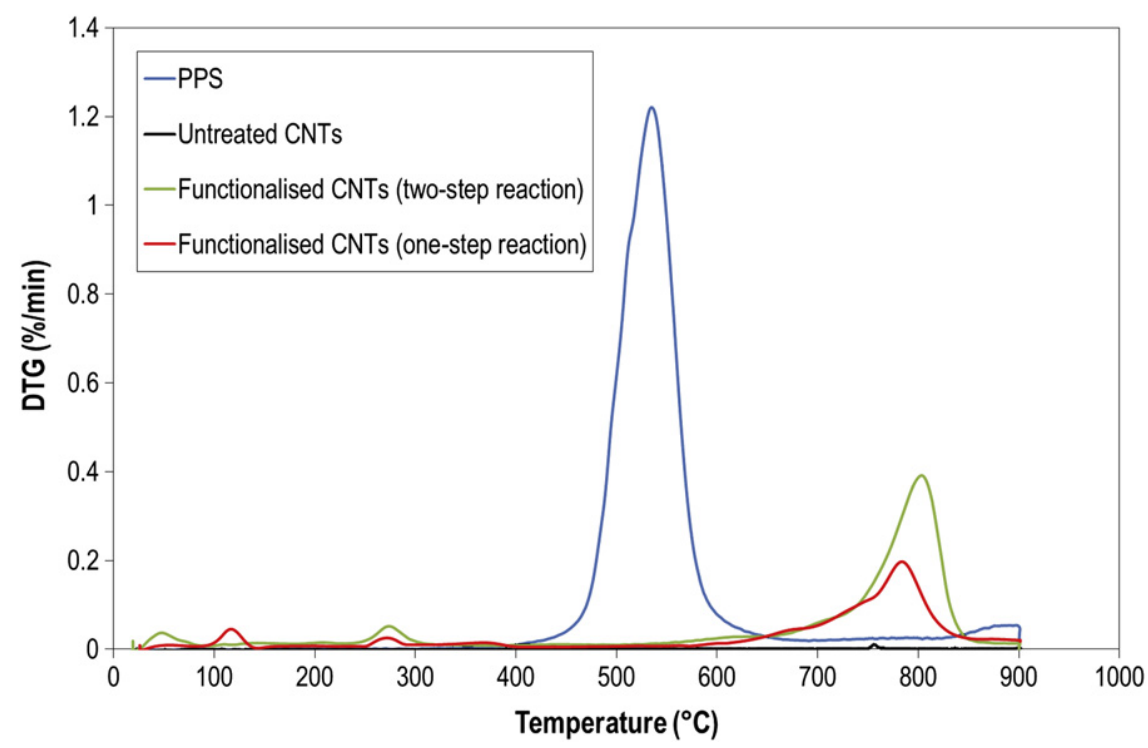

Fig. 10. DTG results of the pure PPS, untreated CNTs and functionalized CNTs in two-step and in a single step reaction.

the loss of polymeric groups physically attached to the surface of CNTs. In contrast, the weight loss at higher temperatures is mainly due to the decomposition of the polymeric groups chemically anchored to the CNT surface. Fig. 10 shows that the thermal stability of PPS is approximately $550{ }^{\circ} \mathrm{C}$, while the grafted chains have higher thermal stability, suggesting the chemical bonding of the oligomers on the surface. The analysis of the CNTs by means of XPS confirmed the formation of covalent bonds between the oligomers and the surface of the CNTs (Gonzalez-Dominguez et al., 2012).

The TGA results in Fig. 9 show a functionalization yield of $30 \mathrm{wt} \%$ and $34 \mathrm{wt} \%$ for one- and two-step reactions, respectively. These values are higher than the yield reported by Gonzalez-Dominguez et al. (2012) for the laboratory scale CNT functionalization (25 wt\%). The higher yields of OFR CNT functionalization were obtained with an initial CNT concentration of $1 \mathrm{wt} \%$ which was far higher than $0.15 \mathrm{wt} \%$ initial concentration which was used in the reported laboratory scale reactions (Gonzalez-Dominguez et al., 2012).

Comparing the yields obtained for the functionalization reactions shows that the yield of the two-step reaction (34 wt\%) was slightly higher than the one-step reaction (30 wt\%); however, the higher yield of the two-step reaction is not large enough to justify the longer processing time as well as large volumes of solvent required for the additional washing and filtration in this process.

In order to confirm the positive effect of the functionalization of CNTs the authors have developed high-performance PPS-based composites through melt mixing (Gonzalez-Dominguez et al., 2012). The obtained nanocomposite materials exhibited a noticeable increase in crystallinity (up to $11 \%$ increase in $\chi c$ ), and thermal stability (up to $17{ }^{\circ} \mathrm{C}$ increase in $T_{\max }$ ), as compared with the PPS matrix, being far better than those exhibited by analogous nanocomposites with bare MWCNTs. These nanocomposites also presented outstanding improvements in mechanical properties, particularly in the tensile ones, exhibiting maximum improvements of nearly $80 \%$ in tensile strength and $100 \%$ in Young's modulus ( $5 \mathrm{wt} \%$ filler loading). The electrical properties of the composites reached maximum values of $\sim 10^{-4} \mathrm{~S} / \mathrm{cm}$ and percolation thresholds below $1 \mathrm{wt} \%$ a clear indication of the positive effect of the functionalization. 


\section{Conclusions}

This paper has described the design and development of a batch oscillatory flow reactor (OFR) which has been used to functionalize CNTs. Oscillation conditions were found where effective and uniform mixing was achieved throughout the reactor and it was discovered that when CNTs were introduced into the liquid dispersion they surprisingly formed a uniform aggregated flocs suspension with an aggregate size in the range of a few microns to $0.5 \mathrm{~mm}$. At this stage, it is unclear why aggregated suspensions were formed. The time averaged uniformity of mixing within the OFR clearly results in a time averaged uniform suspension throughout the OFR, however the reason for aggregation of sub-millimetre and millimetre aggregates is unclear. From the magnitude of the settling velocity after oscillation and also visual observation, it appears that the aggregates themselves are highly voided with an estimated voidage of order $50 \%$. This is useful in relation to chemical activity within the whole aggregate. It might have been expected that more intense oscillation conditions would create smaller aggregates however within the limited range available from the pilot apparatus; no visual difference in flocs size could be seen.

A two or one step functionalization reaction of CNTs with PPS compatible groups was carried out and although the mixing produced CNT aggregates, effective functionalization of the CNT loading was validated using a number of techniques.

Because large flocs of CNT were formed by the OFM, it was discovered that on the cessation of oscillation the CNT flocs would rapidly sediment within the solvent thereby providing an effective separation mechanism and avoid having to use pressure driven ultrafiltration. Functionalization did not appear to change the state of aggregation within the OFR reactor, suggesting that the CNT aggregation was dominantly controlled by mechanical, rather than chemical entanglement.

The final product obtained from the OFR reactor was in the form of CNT aggregates. Whilst this offers certain advantages in terms of mechanical handling; for effective application within a polymer matrix it will be necessary to devise a way of dispersing the CNT within in this case a PPS thermoplastic melt. This may be achieved by using either a melt twin screw extruder or some other form of compounder.

\section{Acknowledgements}

We would like to thank European FP7 project "M-RECT" for the funding of this project and MRECT partners for providing useful background information. In particular we would like to thank Dr. Alan Wood (Victrex plc) for his support of the work. The authors would like to thank Dr. Simon Butler at the Department of Chemical Engineering and Biotechnology at Cambridge University for his assistance. One of us SB would like to thank Trinity College Cambridge for scholarship support.

\section{References}

Ajayan, P.M., Schadler, L.S., Giannaris, C., Rubio, A., 2000. Single-walled carbon nanotube-polymer composites: strength and weakness. Adv. Mater. 12, 750-753.

Bahr, J.L., Tour, J.M., 2001. Highly functionalized carbon nanotubes using in situ generated diazonium compounds. Chem. Mater. 13, 3823-3824.

Brunold, C.R., Hunns, J.C.B., Mackley, M.R., Thompson, J.W., 1989. Experimental observations on flow patterns and energy losses for oscillatory flow in ducts containing sharp edges. Chem. Eng. Sci. 44, 1227-1244.

Calvert, P., 1999. Nanotube composites: a recipe for strength. Nature 399, 210-211.
Coleman, J.N., Khan, U., Blau, W.J., Gun'ko, Y.K., 2006. Small but strong: a review of the mechanical properties of carbon nanotube-polymer composites. Carbon 44, 1624-1652.

Davies, V.A., Ericson, L.M., Parra-Vasquez, A.N.G., Fan, H., Wang, Y., Prieto, V., Longoria, J.A., Ramesh, S., Saini, R.K., Kittrell, C., Billups, W.E., Adams, W.W., Hauge, R.H., Smalley, R.E., Pasquali, M., 2004. Phase behaviour and rheology of SWNTs in superacids. Macromolecules 37, 154-160.

Deng, F., Ogasawara, T., Takeda, N., 2007. Tensile properties at different temperature and observation of micro deformation of carbon nanotubes-poly(ether ether ketone) composites. Compos. Sci. Technol. 67, 2959-2964.

Díez-Pascual, A.M., Guan, J., Simard, B., Gómez-Fatou, M.A., 2011. Poly(phenylene sulphide) and poly(ether ether ketone) composites reinforced with singlewalled carbon nanotube buckypaper: II-Mechanical properties, electrical and thermal conductivity. Composites Part A: Applied Science and Manufacturing, 43, 1007-1015.

Fahey, D.R., Ash, C.E., 1991. Mechanism of poly(para-phenylene sulfide) growth from para-dichlorobenzene and sodium sulfide. Macromolecules 24, 4242-4249.

Gojny, F.H., Wichmann, M.H.G., Kopke, U., Fiedler, B., Schulte, K., 2004. Carbon nanotube-reinforced epoxy composites enhanced stiffness and fracture toughness at low nanotube content. Compos. Sci. Technol. 64, 2363-2371.

Gonzalez-Dominguez, J.M., Castell, P., Bespín-Gascón, S., Ansón-Casaos, A., DíezPascual, A.M., Goméz-Fatou, M.A., Benito, A.M., Maser, W.K., Martinez, M.T., 2012. Covalent functionalization of MWCNTs with poly(p-phenylene sulphide) oligomers chains: a route towards the efficient integration through a chemical approach. J. Mater. Chem. , http://dx.doi.org/10.1039/C2JM35272A.

Harvey, A.P., Mackley, M.R., Stonestreet, P., 2001. Operation and optimization of an oscillatory flow continuous reactor. Ind. Eng. Chem. Res. 40, 5371-5377.

Howes, T., 1988. On the dispersion of unsteady flow in baffled tubes. Ph.D. Thesis. University of Cambridge.

Huang, Y.Y., Ahir, S.V., Terentjev, E.M., 2006. Dispersion rheology of carbon nanotubes in a polymer matrix. Phys. Rev. B 73, 125422.

Iijima, S., 1991. Helical microtubules of graphitic carbon. Nature 354, 56-58.

Jin, L., Bower, C., Zhou, O., 1998. Alignment of carbon nanotubes in polymer matrix by mechanical stretching. Appl. Phys. Lett 73, 1197-1199.

Kim, Y.J., Shin, T.S., Choi, H.D., Kwon, J.H., Chung, Y.C., Yoon, H.G., 2005. Electrical conductivity of chemically modified multi-walled carbon nanotube/epoxy composites. Carbon 43, 23-30.

Lau, K., Gu, C., Hui, D., 2006. A critical review on nanotube and nanotube/nanoclay related polymer composite materials. Composites Part B 37, 425-436.

Neves-Saraiva, R.M.D.C., 1998. The characterisation of mixing for oscillatory flow within baffled tubes. Ph.D. Thesis. University of Cambridge.

Ni, X., Mackley, M.R., 1993. Chemical reaction in batch pulsatile flow and stirred tank reactor. Chem. Eng. J. 52, 107-114.

Nienow, A.W., 2010. Stirred tank reactors. Ullmann's Encyclopedia of Industrial Chemistry.

Park, L.S., Kim, G.H., Lee, S.C., Han, S.K., Cha, I.H., 1992. Polymerization and copolymerization of poly(p-phenylene sulfide) and poly( $p$-phenylene sulfide/ sulfone). Polym. Korea 16, 687-692.

Perry, 1984. Chemical Engineer's Handbook, sixth ed. McGraw-Hill, New York.

Platt, D.K., 2003. Engineering and high performance plastics. Rapra Technology Ltd, Shawbury 23-24.

Qiam, D., Dickey, E.C., Andrews, R., Rantell, T., 2000. Load transfer and deformation mechanisms in carbon nanotube-polystyrene composites. Appl. Phys. Lett. 76, 2868-2870.

Ramamurthy, P.C., Malshe, A.M., Harrell, W.R., Gregory, R.V., McGuire, K., Rao, A.M., 2004. Polyaniline/single-wall carbon nanotube composite electronic devices. Solid State Electron 48, 2019-2024.

Saito, S., 1997. Carbon nanotubes for next-generation electronic devices. Science 278, 77-78.

Shao, L.H., Luo, R.Y., Bai, S.L., Wang, J., 2009. Prediction of effective moduli of carbon nanotube-reinforced composites with waviness and debonding. Compos. Struct. 87, 274-281.

Smith, K.B., 1999. The scale-up of oscillatory flow mixing. Ph.D. Thesis. University of Cambridge.

Smith, K.B., Mackley, M.R., 2006. An experimental investigation into the scale-up of oscillatory flow mixing in baffled tubes. Trans IChemE, Part A 84 (A11), 1001-1011.

Tans, S.J., Verschueren, A.R.M., Dekker, C., 1998. Room-temperature transistor based on a single carbon nanotube. Nature 393, 49-52.

Tasis, D., Tagmatarchis, N., Bianco, A., Prato, M., 2006. Chemistry of carbon nanotubes. Chem. Rev. 106, 1105-1136.

Thostenson, E.T., Chow, T.W., 2002. Aligned multi-walled carbon nanotubereinforced composites processing and mechanical characterization. J. Phys. D: Appl. Phys 35, L77-L80.

Tsuchida, E., Kimihisa, Y., Hiroyuki, N., Shu, Y., Mitsutoshi, J., 1990. Polymerization of diphenyl disulphide by the S-S bond cleavage with a Lewis acid. A novel preparation route to poly( $p$-phenylene sulphide). Macromolecules 23, 2101-2106.

Vaisman, L., Wagner, H.D., Marom, G., 2006. The role of surfactants in dispersion of carbon nanotubes. Adv. Colloid Interface Sci. 128-130, 37-46.

Wood, J.R., Zhao, Q., Wagner, H.D., 2001. Orientation of carbon nanotubes in polymers its detection by Raman spectroscopy. Composites Part A 32, 391-399.

Xie, X.L., Mai, Y.W., Zhow, X.P., 2005. Dispersion and alignment of carbon nanotubes in polymer matrix: a review. Mater. Sci. Eng., R 49, 89-112. 